\title{
Comparative Studies on the Interaction of Rhodamine B with Bovine Serum Albumin Using Fluorescence Method and Synchronous Fluorescence Method
}

\author{
Chundan Wang, Baosheng Liu*, Lihua Ma, Hongcai Zhang, Xu Cheng \\ College of Chemistry \& Environmental Science, Hebei University, Baoding, P. R. China \\ Email address: \\ lbs@hbu.edu.cn (Baosheng Liu) \\ ${ }^{*}$ Corresponding author

\section{To cite this article:} \\ Chundan Wang, Baosheng Liu, Lihua Ma, Hongcai Zhang, Xu Cheng. Comparative Studies on the Interaction of Rhodamine B with Bovine \\ Serum Albumin Using Fluorescence Method and Synchronous Fluorescence Method. Journal of Chemical, Environmental and Biological \\ Engineering. Vol. 2, No. 1, 2018, pp. 17-25. doi: 10.11648/j.jcebe.20180201.14
}

Received: May 4, 2018; Accepted: June 6, 2018; Published: July 4, 2018

\begin{abstract}
The reaction mechanism of rhodamine B (RHB) with bovine serum albumin (BSA) was investigated using fluorescence spectroscopy and synchronous fluorescence spectroscopy at different temperatures (298 K, 310 K and $318 \mathrm{~K})$. The results showed that electrostatic force played a major role on the conjugation reaction between BSA and RHB, and the type of quenching was static quenching. Primary binding site for RHB was sub-hydrophobic domain IIA, and the number of binding sites was 1 . The order of magnitude of binding constants $\left(K_{a}\right)$ was $10^{4}$. The value of Hill's coefficients $\left(n_{H}\right)$ was approximately equal to 1, which suggested no cooperativity in BSA-RHB system. The donor-to-acceptor distance $r<7 \mathrm{~nm}$ indicated that the static fluorescence quenching of BSA by RHB was also a non-radiation energy transfer process. The results of two methods were consistent that showed the synchronous fluorescence spectroscopy could be used to study the reaction mechanism between drug and protein, and was a useful supplement to the conventional fluorescence quenching method.
\end{abstract}

Keywords: Fluorescence Spectroscopy, Synchronous Fluorescence Spectroscopy, Rhodamine B, Bovine Serum Albumin, Reaction Mechanism

\section{Introduction}

RHB is a strong fluorescence of synthetic dyes, and is used widely in biotechnology [1], printing [2], indicator of trace detection [3], etc. Because of the bright color, strong stability and low price etc., RHB was added to food as food additives by some illegal businessmen, such as pepper products, bean products, condiment, etc. But a growing number of studies have shown that RHB has the potential of carcinogenicity. An assessment about chemicals causing cancer risk was made by the International Agency for Research on Cancer (IARC) showed that ingestion, inhalation and skin contact of RHB will cause harm to acute or chronic poisoning. As a result, European Union (EU) issued corresponding documents for banning people from adding RHB to the food. In 2008, the Ministry of Health in China added RHB to the list (first) what might be illegal or non-food substances and abusive food additives varieties in the food, and prohibited RHB using in the food. However, there are still some illegal businessmen who covet interests and add RHB into food as food additives, which bring diet safety hidden trouble for people.

Serum albumin is the most abundant protein as important carrier in plasma, and can interact with many endogenous and exogenous substances. The research between small molecules and serum albumin can make us know their properties of forces and interaction mechanism at molecular level. Traditional fluorescence is that the interaction between small molecules and protein occur leading to the decrease of the intrinsic fluorescence intensity of BSA. Synchronous fluorescence spectroscopy was excitation and emission monochromators are scanned simultaneously to study the reaction mechanism between bovine serum albumin and substances. Comparing synchronous fluorescence spectroscopy and conventional fluorescence spectroscopy, synchronous fluorescence spectrometry shows good selectivity, high sensitivity, simplified spectrum, narrowed 
bands and less interference etc $[4,5]$. Fluorescence quenching method is a commonly used method for studying the reaction mechanism of substances with bovine serum albumin, but the research by synchronous fluorescence method is less reported. In this paper, under the simulated physiological conditions, the interaction between RHB and BSA has been studied by fluorescence quenching spectroscopy and synchronous fluorescence spectroscopy. The research provides the help for understanding the toxicity of RHB, and urging people to improve the consciousness of food safety and ensure food safety.

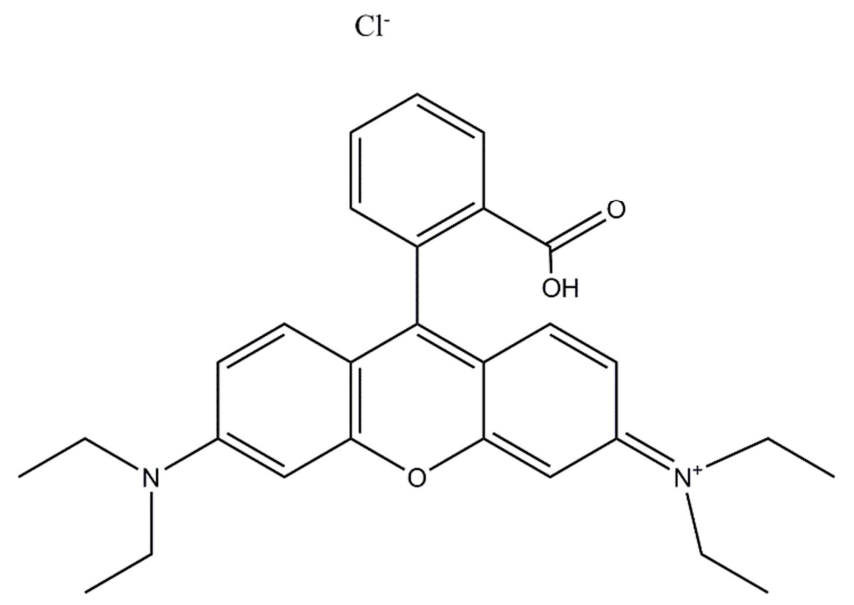

Figure 1. Chemical structure of Rhodamine B.

\section{Experimental}

\subsection{Reagents and Chemicals}

Bovine serum albumin (BSA) was purchased from Sigma Co. and was of the purity grade inferior 99\%. RHB was purchased from the third factory of Shanghai reagent. Stock solutions of BSA $\left(1.0 \times 10^{-4} \mathrm{M}\right)$ and RHB $\left(1.0 \times 10^{-4} \mathrm{M}\right)$ were prepared. All the stock solutions were further diluted for use as working solutions. Tris-HCl buffer solution containing 0.15 $\mathrm{M} \mathrm{NaCl}$ was used to maintain the $\mathrm{pH}$ of solutions at 7.40 and $\mathrm{NaCl}$ solution was used to maintain the ionic strength of the solution. All other reagents were of analytical grade and all aqueous solutions were prepared with newly double-distilled water and stored at $277 \mathrm{~K}$.

\subsection{Apparatus}

All fluorescence spectra were recorded with a Shimadzu RF-5301PC spectrofluorophotometer. Absorption was measured with an UV-vis recording spectrophotometer (UV-265, Shimadzu, Japan). All $\mathrm{pH}$ measurements were carried out with a pHS-3C precision acidity meter (Leici, Shanghai, China). All temperatures were controlled by a SYC-15 1 superheated water bath (Nanjing Sangli Electronic Equipment Factory).

\subsection{Procedures}

\subsubsection{Fluorescence Measurements}

In a typical fluorescence measurement, $1.0 \mathrm{~mL}$ of Tris- $\mathrm{HCl}$ solution, $1.0 \mathrm{~mL}$ of $2.0 \times 10^{-6} \mathrm{M}$ BSA solution and different concentrations of RHB were successively added to a $10 \mathrm{~mL}$ colorimetric tube. The samples were diluted to scaled volume with water, mixed thoroughly by shaking, and kept static for $30 \mathrm{~min}$ at different temperatures $(298 \mathrm{~K}, 310 \mathrm{~K}$ and $318 \mathrm{~K})$. The excitation and emission slits were set at $5 \mathrm{~nm}$. The excitation wavelength for BSA was $280 \mathrm{~nm}$ and $295 \mathrm{~nm}$, respectively, with a $10 \mathrm{~nm}$ path length cell. We recorded the intensity of fluorescence at $340 \mathrm{~nm}$.

\subsubsection{Synchronous Fluorescence Measurements}

Solution preparation was as detailed above. The difference between excitation wavelength and emission wavelength $\Delta \lambda$ was set at $15 \mathrm{~nm}$ and $60 \mathrm{~nm}$, respectively. Then we recorded the intensity of synchronous fluorescence at $300 \mathrm{~nm}$ and 340 $\mathrm{nm}$, respectively.

\subsubsection{UV-vis Measurements}

$1.0 \mathrm{~mL}$ of Tris- $\mathrm{HCl}$ solution, $1.0 \mathrm{~mL}$ of $1.0 \times 10^{-4} \mathrm{M}$ BSA solution and different concentrations of RHB were successively added to a $10 \mathrm{~mL}$ colorimetric tube, and the reference solutions were the corresponding concentration of RHB solutions. The samples were diluted to scaled volume with water, mixed thoroughly by shaking, and kept static for $30 \mathrm{~min}$ at $298 \mathrm{~K}$. The UV-vis absorption spectrum of RHB in the presence and absence of BSA were scanned with $1 \mathrm{~cm}$ quartz cells over the range from 190 to $300 \mathrm{~nm}$.

\section{Results and Discussion}

\subsection{UV-vis Absorption Spectra Studie}

BSA has two absorption peaks, which is at $225 \mathrm{~nm}$ and at $275 \mathrm{~nm}$. The absorption peak at $225 \mathrm{~nm}$ which is mainly caused by $n-\pi *$ transition of $\mathrm{C}=\mathrm{O}$ of peptide bonds, and is related to the content of $\alpha$ - helical in BSA. Absorption peak at $275 \mathrm{~nm}$ is caused by $\pi-\pi^{*}$ and $n-\pi^{*}$ transition of heterocyclic aromatic of two tryptophans and nineteen tyrosines in BSA molecule [6]. With the increasing concentration of RHB, Figure 2 show that the intensity of absorption peak at $225 \mathrm{~nm}$ decreases with red shift $5 \mathrm{~nm}$. The results suggest that the interaction between RHB and BSA resulted in the formation of a new complex, and the content of $\alpha$ - helix was changed resulting in conformational changes of BSA. The intensity of the absorption peak at $275 \mathrm{~nm}$ was reduced, illustrating that the interaction between RHB and BSA happened in the ground-state molecules. That is to say, non-luminous complex formed in ground state of RHB and BSA, which led to the change of Uv-vis absorption spectra of BSA [7]. Dynamic quenching only affects the excited states of fluorescent molecule, and does not alter the absorption spectra of the fluorescent substance [8]. So we can get preliminary inference that type of fluorescence quenching of RHB with BSA was static quenching. 


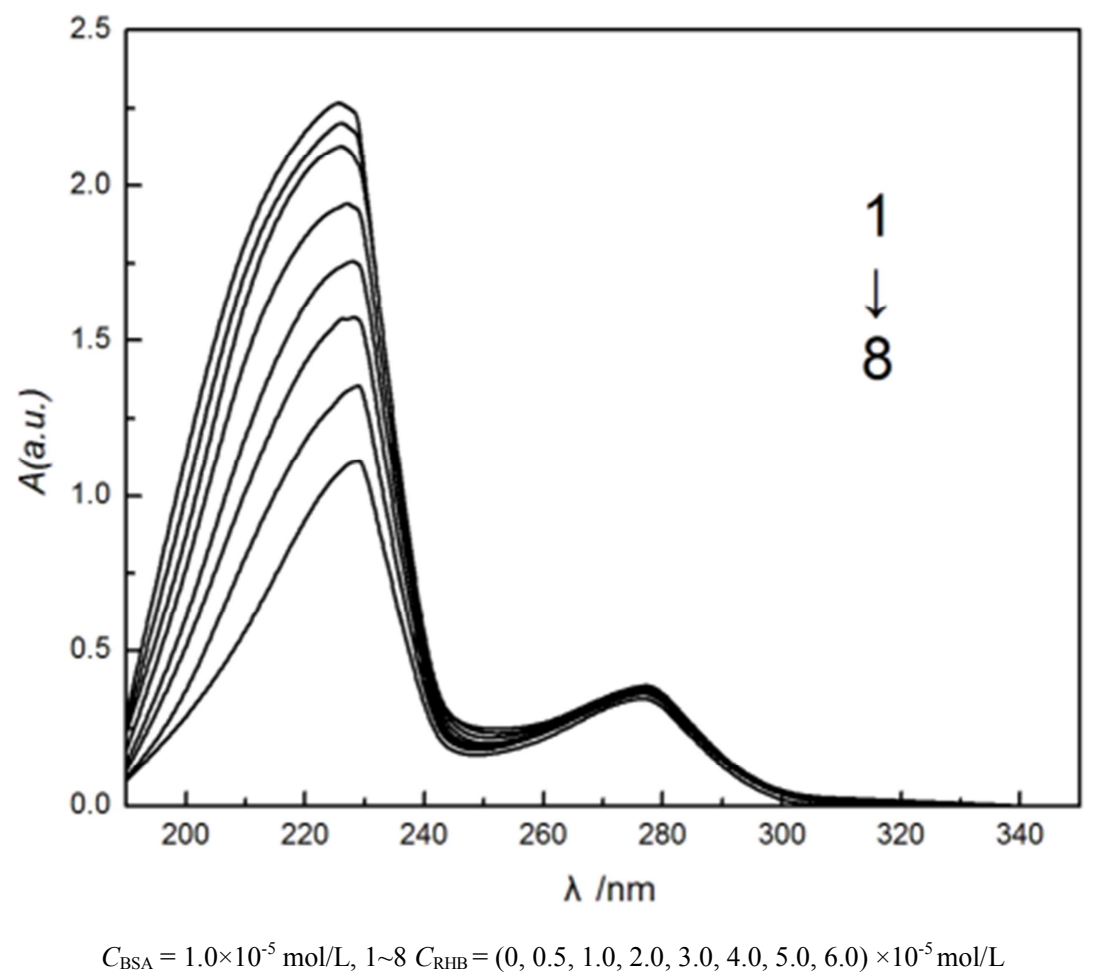

Figure 2. UV absorption spectra of BSA-RHB system $(T=298 \mathrm{~K})$.

\subsection{Fluorescence Quenching Spectra of BSA-RHB System}

The fluorescence spectra of BSA-RHB system were shown in Figure 3 (similar to $295 \mathrm{~nm}$ ). As shown in Figure 3, the fluorescence intensity of BSA decreased regularly with the addition of RHB when the excitation wavelength was $280 \mathrm{~nm}$, which indicated that RHB could quench the intrinsic fluorescence of BSA strongly and there was an interaction between RHB and BSA.

In order to confirm the quenching mechanism, the fluorescence quenching data were analyzed by the Stern-Volmer Eq. (1) [9]:

$$
F_{0} / F=1+K_{q} \tau_{0}[L]=1+K_{s v}[L]
$$

Where, $F_{0}$ and $F$ represent the fluorescence intensities in the absence and presence of quencher, respectively. $\tau_{0}$ is the average lifetime of the fluorescence without quencher, and is $10^{-8} \mathrm{~s}[10] . K_{s v}$ is the Stern-Volmer quenching constant. $K_{q}$ is the bimolecular quenching constant and $[L]$ is the concentration of the quencher. Based on the linear fit plot of $F_{0} / F$ vs. [L], values of $K_{s v}$ and $K_{q}$ could be obtained at different temperatures. The calculated results were shown in Table 1. Table 1 showed that the value of $K_{s v}$ gradually decreased with rising temperature. That is to say, the extent of fluorescence quenching of RHB to BSA was reduced with rising temperature. The results indicated that fluorescence quenching was not caused by diffusion and collision of dynamic quenching, but was static quenching process forming ground-state complex between RHB and BSA [11]. In addition, values of $K_{q}$ were much greater than the maximum scatter collision quenching constant of various quenchers $\left(2 \times 10^{10} \mathrm{M}^{-1} \cdot \mathrm{s}^{-1}\right)$ [12] under different temperatures, which also suggested that the quenching was a static process [13]. This conclusion is consistent with the Uv-vis absorption spectra.

Table 1. Quenching reactive parameters of BSA and RHB at different temperatures.

\begin{tabular}{llllllll}
\hline$\lambda_{\text {ex }} /(\mathbf{n m})$ & $\boldsymbol{T} / \mathbf{K}$ & $\boldsymbol{K}_{\mathbf{q}} /\left(\mathbf{L} \cdot \mathbf{m o l}^{-1} \cdot \mathbf{s}^{-1}\right)$ & $\boldsymbol{K}_{\mathrm{sv}} /\left(\mathbf{L} \cdot \mathbf{m o l}^{-1}\right)$ & $\boldsymbol{r}_{\mathbf{1}}$ & $\boldsymbol{K}_{\mathbf{a}} /\left(\mathbf{L} \cdot \mathbf{m o l}^{-1}\right)$ & $\boldsymbol{n}$ & $\boldsymbol{r}_{\mathbf{2}}$ \\
\hline \multirow{3}{*}{280} & 298 & $6.82 \times 10^{12}$ & $6.82 \times 10^{4}$ & 0.9929 & $6.73 \times 10^{4}$ & 1.06 & 0.9947 \\
& 310 & $6.34 \times 10^{12}$ & $6.34 \times 10^{4}$ & 0.9949 & $6.47 \times 10^{4}$ & 0.83 & 0.9939 \\
& 318 & $6.29 \times 10^{12}$ & $6.29 \times 10^{4}$ & 0.9973 & $6.25 \times 10^{4}$ & 0.78 & 0.9993 \\
295 & 298 & $5.87 \times 10^{12}$ & $5.87 \times 10^{4}$ & 0.9926 & $5.44 \times 10^{4}$ & 0.99 & 0.9961 \\
& 310 & $5.84 \times 10^{12}$ & $5.84 \times 10^{4}$ & 0.9957 & $5.13 \times 10^{4}$ & 0.79 & 0.9985 \\
& 318 & $5.46 \times 10^{12}$ & $5.46 \times 10^{4}$ & 0.9996 & $5.09 \times 10^{4}$ & 0.79 & 0.9996 \\
\hline
\end{tabular}

$K_{\mathrm{q}}$ is the quenching rate constant. $r_{1}$ is the linear relative coefficient of $F_{0} / F \sim[L] . K_{\mathrm{a}}$ is the binding constant. $n$ is the number of binding site. $r_{2}$ is the linear relative coefficient of $\log \left(F_{0}-F\right) / F \sim \log \left\{\left[D_{t}\right]-n\left[B_{t}\right]\left(F_{0}-F\right) / F_{0}\right\}$. 


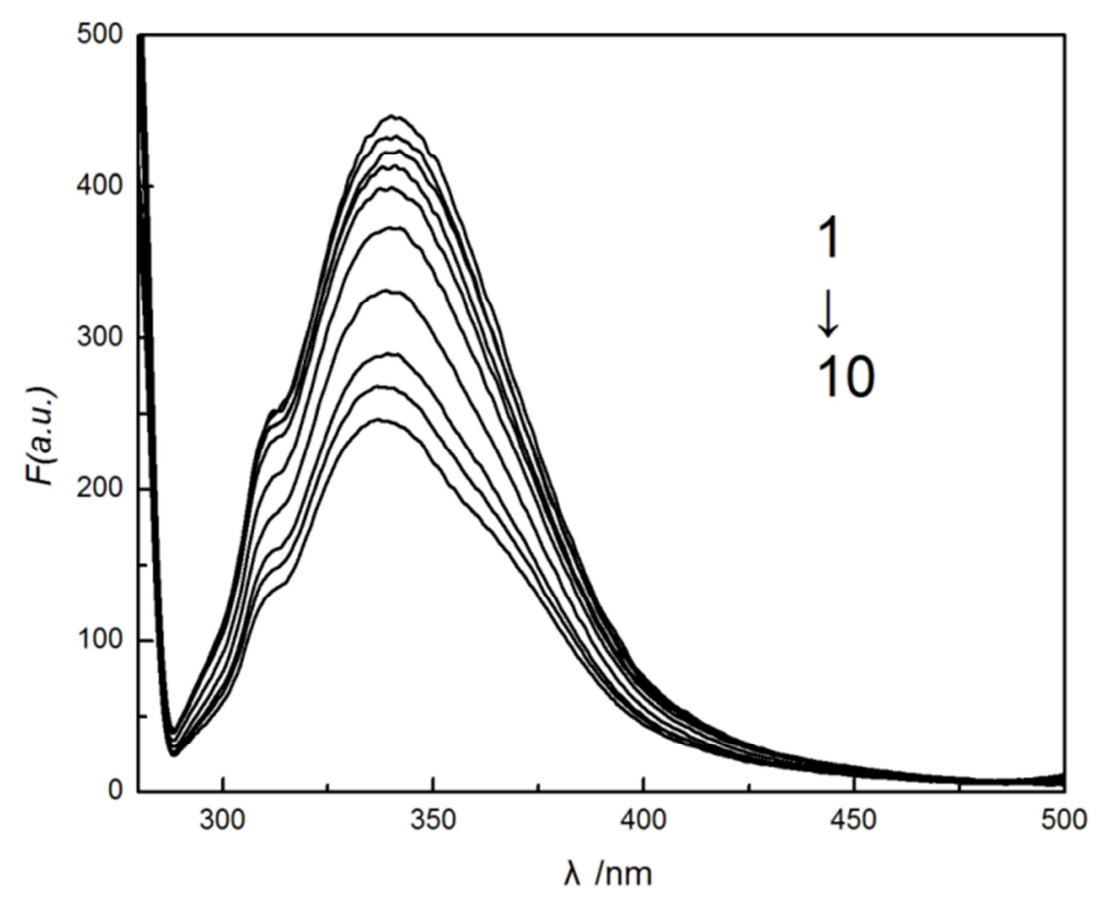

$C_{\mathrm{BSA}}=2.0 \times 10^{-7} \mathrm{~mol} / \mathrm{L}, 1 \sim 10 C_{R H B}=(0,0.1,0.5,1.0,2.0,4.0,6.0,8.0,10.0,12.0) \times 10^{-6} \mathrm{~mol} / \mathrm{L}$

Figure 3. Fluorescence spectra of BSA-RHB B system $\left(T=298 \mathrm{~K}, \lambda_{e x}=280 \mathrm{~nm}\right)$.

For static quenching process, the relationship between the fluorescence intensity and the concentration of quencher can be usually described by derived Eq. (2) [14] to obtain the binding constant $\left(K_{a}\right)$ and the number of binging sites $(n)$ in most papers:

$$
\lg \left(\frac{F_{0}-F}{F}\right)=n \lg K_{a}+\mathrm{n} \lg \left\{\left[D_{t}\right]-n \frac{F_{0}-F}{F_{0}}\left[B_{t}\right]\right\}
$$

Where $\left[D_{t}\right]$ and $\left[B_{t}\right]$ are the total concentrations of RHB and BSA, respectively. On the assumption that $n$ in the bracket is equal to 1 (the results remain the same no matter what the value of $n$ is hypothesized in the bracket, for example, $n=1,2,3,7.3$ or 13.7 , the calculation remain the same). The curve of $\log \left[\left(F_{0}-F\right) / F\right]$ versus $\log$ $\left\{\left[D_{t}\right]-\left[B_{t}\right]\left(F_{0}-F\right) / F_{0}\right\}$ is drawn and fitted linearly, then the value of $n$ can be obtained from the slope of the plot. If the $n$ value obtained is not equal to 1 , then it is substituted into the bracket and the curve of $\log \left(F_{0}-F\right) / F$ versus $\log \left\{\left[D_{t}\right]-n\right.$ $\left.\left[B_{t}\right]\left(\mathrm{F}_{0}-\mathrm{F}\right) / \mathrm{F}_{0}\right\}$ is drawn again. The above process is repeated again and again till $n$ obtained is only a single value or a circulating value. The value of $n$ and binding constant $K_{a}$ can be obtained from the slope and the intercept of the plot which were shown in Table 1. As seen in Table 1, values of $n$ were all about 1, which suggested that just one binding site for RHB existed in BSA. Meanwhile, the binding constants $K_{a}$ decreased with increasing temperature, that is to say, the stability of ground-state complex was reduced with rising temperature, further suggesting that the quenching was a static process [15].

\subsection{The Primary Binding Site Studies}

At $280 \mathrm{~nm}$ wavelength, the tryptophan residues and tyrosine residues in BSA are excited, whereas a wavelength of $295 \mathrm{~nm}$ excites only tryptophan residues. In BSA, sub-hydrophobic domain IIA (containing both tryptophan and tyrosine) and IIIA (containing only tyrosine) are the main binding sites for small molecule ligands [16]. Comparing the fluorescence quenching of BSA excited at $280 \mathrm{~nm}$ and $295 \mathrm{~nm}$ allows us to estimate the participation of tryptophan and tyrosine groups in the system, and to determine its specific location [17]. As shown in Figure 4, fluorescence quenching curve of BSA excited at $280 \mathrm{~nm}$ and $295 \mathrm{~nm}$ did not overlap, and the extent of fluorescence quenching at $280 \mathrm{~nm}$ was larger than the extent of fluorescence quenching at $295 \mathrm{~nm}$, which suggested that both tryptophan and tyrosine participated in the reaction of RHB with BSA. Therefore, it might be implied that the primary binding site for RHB was sub-hydrophobic domain IIA. 


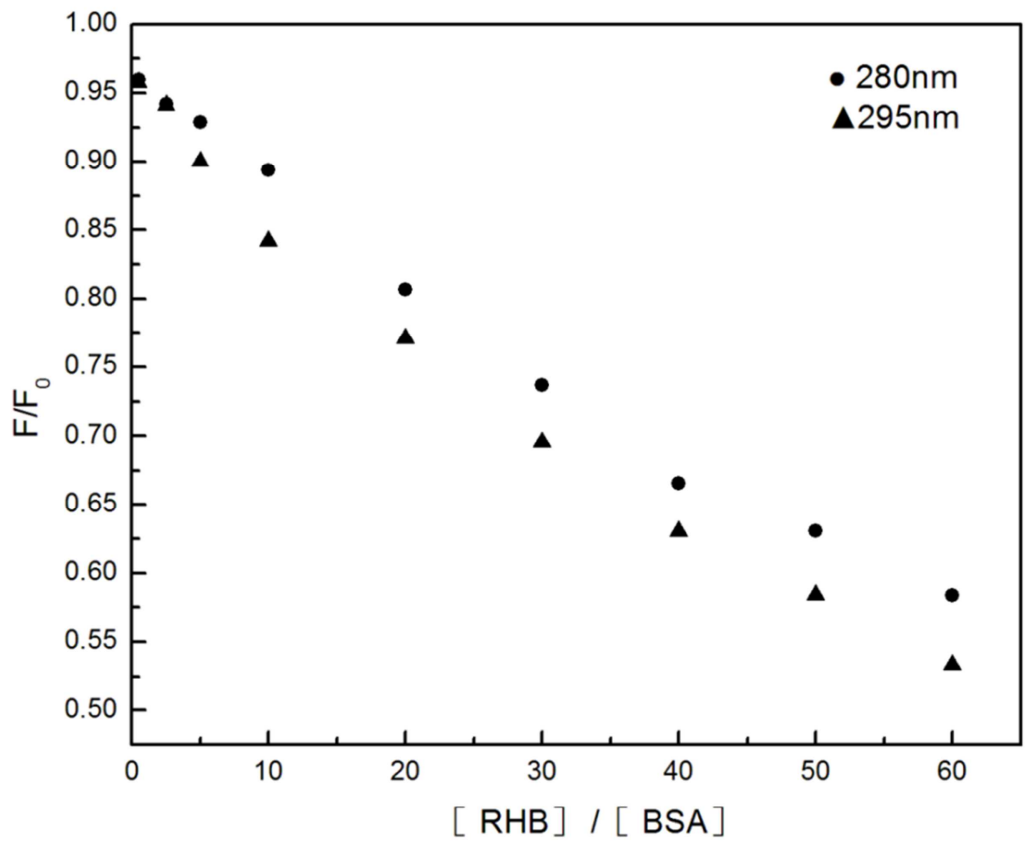

$C_{\mathrm{BSA}}=2.0 \times 10^{-7} \mathrm{~mol} / \mathrm{L} ; C_{\mathrm{RHB}}=(0,0.1,0.5,1.0,2.0,4.0,6.0,8.0,10.0,12.0) \times 10^{-6} \mathrm{~mol} / \mathrm{L}$

Figure 4. Quenching curves of BSA-RHB system at $\lambda_{e x}=280 \mathrm{~nm}$ and $295 \mathrm{~nm}(T=298 \mathrm{~K})$.

\subsection{Synchronous Fluorescence Spectra Studies}

When the value of $\Delta \lambda$ between the excitation and emission wavelengths is stabilized at either $15 \mathrm{~nm}$ or $60 \mathrm{~nm}$, synchronous fluorescence gives characteristic information for tyrosine or tryptophan residues, respectively [18]. The location change of maximum emission wavelength of synchronous fluorescence can reflect the change of polarity of group molecules [19]. It could be seen in the Figure 5 that when $\Delta \lambda$ was $15 \mathrm{~nm}$, the fluorescence intensities of BSA-RHB showed obvious decrease, and had no shifts with increasing the concentration of RHB. When $\Delta \lambda$ was $60 \mathrm{~nm}$, the synchronous fluorescence intensities of BSA-RHB decreased regularly with blue shifts, which indicated that the microenvironment of tryptophan was changed due to the interaction of RHB with BSA, and the polarity of the hydrophobic environment was reduced and hydrophobicity was enhanced in the BSA cavity due to changes in the microenvironment of the tryptophan residues on insertion of RHB [20]. This led to the conformational changes of BSA. When the value of $\Delta \lambda$ between the excitation and emission wavelengths was stabilized at either 15 or $60 \mathrm{~nm}$, the synchronous fluorescence intensity was reduced, which further suggested the tryptophan and tyrosine were involved in the interaction of BSA and RHB. For the quenching process, we calculated the combinational parameters of system using equation (1), (2), and the results were listed in Table 2. As seen in Table 2, the Stern-Volmer quenching constant $K_{s \mathrm{~V}}$ decreased with the increase of temperature, indicating that the quenching process was static quenching. At the same time, the values of $K_{q}$ were much greater than the maximum scatter collision quenching constant of various quenchers $\left(2 \times 10^{10} \mathrm{M}^{-1} \cdot \mathrm{s}^{-1}\right)$ under different temperatures, which proved that the quenching process was a static quenching process with the formation of a new complex. The number of binding bites $(n)$ was about 1 , indicating that one molecule of RHB combines with one molecule of BSA. With increasing temperature, the values of $K_{a}$ were reduced, which suggested the stability of ground-state complex was reduced with increasing temperature and also showed that quenching process was a static quenching process. The quenching mechanism obtained by synchronous fluorescence method was coincident with that obtained by fluorescence quenching method. The change tendency was the same as the excitation wavelength $280 \mathrm{~nm}$. Meanwhile, comparing Table 1and Table 2, you could see that the quenching parameters of BSA-RHB by two methods were in the same order of magnitude.

Table 2. Quenching reactive parameters of RHB and BSA at different temperatures.

\begin{tabular}{|c|c|c|c|c|c|c|c|}
\hline$\Delta \lambda /(\mathbf{n m})$ & $T / K$ & $K_{\mathrm{q}} /\left(\mathrm{L} \cdot \mathrm{mol}^{-1} \cdot \mathrm{s}^{-1}\right)$ & $K_{\mathrm{sv}} /\left(\mathbf{L} \cdot \mathbf{m o l}^{-1}\right)$ & $r_{3}$ & $K_{\mathrm{a}} /\left(\mathrm{L} \cdot \mathrm{mol}^{-1}\right)$ & $n$ & $r_{4}$ \\
\hline \multirow{3}{*}{15} & 298 & $7.75 \times 10^{12}$ & $7.75 \times 10^{4}$ & 0.9957 & $7.76 \times 10^{4}$ & 1.14 & 0.9987 \\
\hline & 310 & $7.36 \times 10^{12}$ & $7.36 \times 10^{4}$ & 0.9982 & $7.36 \times 10^{4}$ & 1.06 & 0.9993 \\
\hline & 318 & $6.98 \times 10^{12}$ & $6.98 \times 10^{4}$ & 0.9992 & $7.14 \times 10^{4}$ & 1.11 & 0.9984 \\
\hline \multirow{3}{*}{60} & 298 & $7.73 \times 10^{12}$ & $7.73 \times 10^{4}$ & 0.9969 & $7.38 \times 10^{4}$ & 0.85 & 0.9973 \\
\hline & 310 & $7.47 \times 10^{12}$ & $7.47 \times 10^{4}$ & 0.9967 & $7.02 \times 10^{4}$ & 0.89 & 0.9986 \\
\hline & 318 & $7.19 \times 10^{12}$ & $7.19 \times 10^{4}$ & 0.9988 & $6.98 \times 10^{4}$ & 0.91 & 0.9959 \\
\hline
\end{tabular}

$K_{\mathrm{q}}$ is the quenching rate constant. $r_{3}$ is the linear relative coefficient of $F_{0} / F \sim[L] . K_{\mathrm{a}}$ is the binding constant. $n$ is the number of binding site. $r_{4}$ is the linear relative coefficient of $\log \left(F_{0}-F\right) / F \sim \log \left\{\left[D_{t}\right]-n\left[B_{t}\right]\left(F_{0}-F\right) / F_{0}\right\}$. 

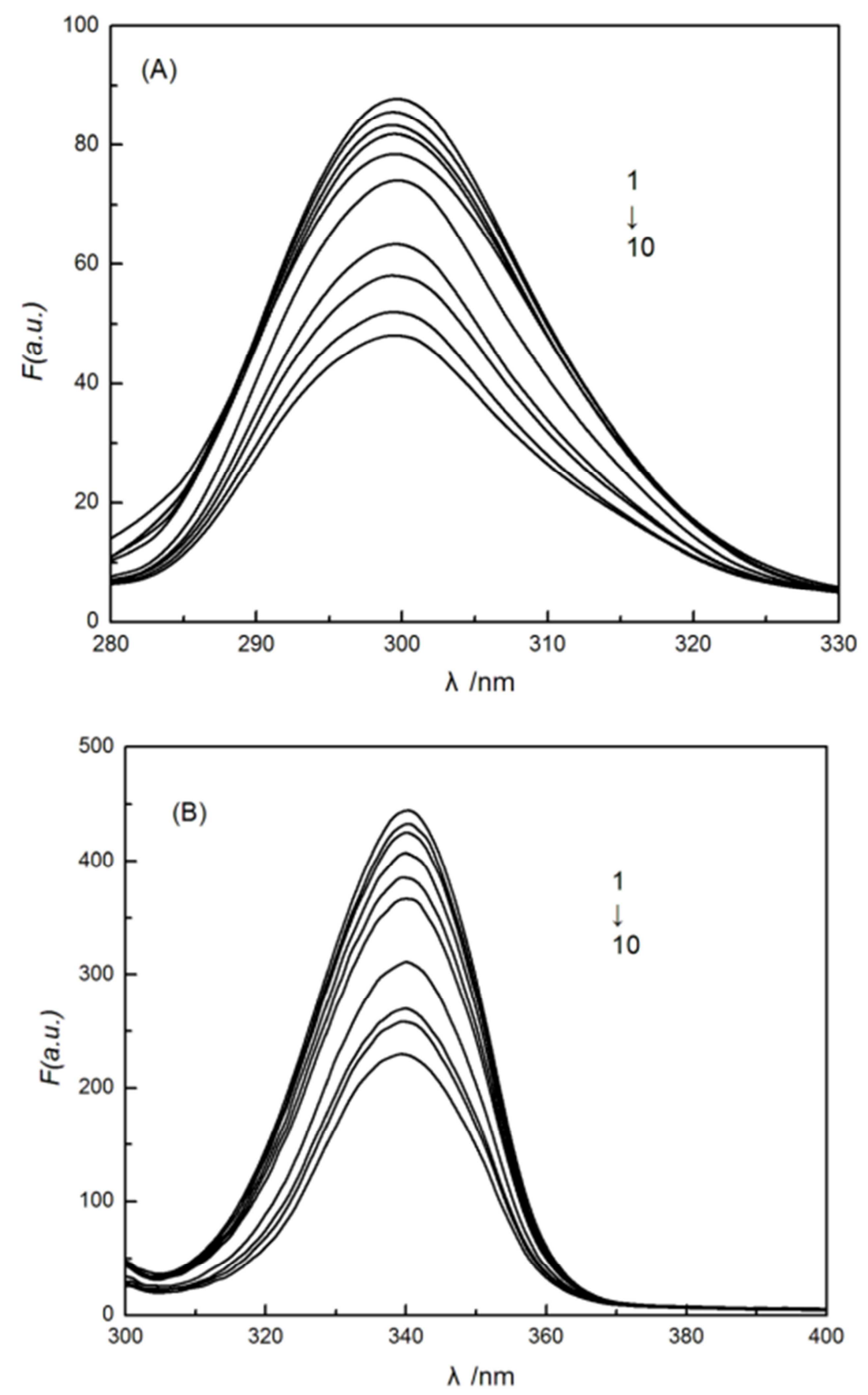

$C_{\mathrm{BSA}}=2.0 \times 10^{-7} \mathrm{~mol} / \mathrm{L}, 1 \sim 10 C_{R H B}=(0,0.1,0.5,1.0,2.0,4.0,6.0,8.0,10.0,12.0) \times 10^{-6} \mathrm{~mol} / \mathrm{L}$

Figure 5. Synchronous fluorescence spectra of BSA-RHB system $(A) \Delta \lambda=15 \mathrm{~nm} ;(B) \Delta \lambda=60 \mathrm{~nm}$.

\subsection{Type of Interaction Force of BSA-RHB System}

The type of interaction force of BSA-RHB systems can be gained through the thermodynamic parameters of reaction of RHB with BSA [21]. If the temperature changes little, the value of $\Delta H$ is regarded as a constant. According to the values of $K_{a}$ of BSA-RHB system at different temperatures from Table 1 and Table 2, the thermodynamic parameters can be calculated on the basis of Eq. (3) and (4):

$$
R \ln K=\Delta S-\Delta H / T
$$

$$
\Delta G=\Delta H-T \Delta S
$$

The results were shown in Table 3. The negative value of $\Delta G$ clarified a spontaneous reaction between BSA and RHB. The negative value of $\Delta H$ and positive value of $\Delta \mathrm{S}$ showed that RHB mainly bound to BSA by the electrostatic attraction [22]. As seen in Table 3, the conclusions drawn from the synchronous fluorescence method were consistent with the fluorescence quenching method, which suggested that synchronous fluorescence method could replace the fluorescence quenching method to determine the type of interaction force of BSA-RHB system. 
Table 3. The thermodynamic parameters of BSA-RHB system at different temperatures.

\begin{tabular}{|c|c|c|c|c|c|}
\hline System & $T /(\mathbf{K})$ & $K a /\left(\mathbf{L} \cdot \mathrm{mol}^{-1}\right)$ & $\Delta H /\left(\mathrm{KJ} \cdot \mathrm{mol}^{-1}\right)$ & $\Delta S /\left(\mathrm{J} \cdot \mathrm{mol}^{-1} \cdot \mathrm{K}^{-1}\right)$ & $\Delta G /\left(\mathrm{KJ}^{\prime} \mathrm{mol}^{-1}\right)$ \\
\hline \multirow{3}{*}{$\Delta \lambda=15 \mathrm{~nm}$} & 298 & $7.76 \times 10^{4}$ & \multirow{3}{*}{-3.16} & 82.99 & -27.90 \\
\hline & 310 & $7.36 \times 10^{4}$ & & 82.96 & -28.89 \\
\hline & 318 & $7.14 \times 10^{4}$ & & 82.97 & -29.55 \\
\hline \multirow{3}{*}{$\Delta \lambda=60 \mathrm{~nm}$} & 298 & $7.38 \times 10^{4}$ & \multirow{3}{*}{-2.49} & 84.81 & -27.77 \\
\hline & 310 & $7.02 \times 10^{4}$ & & 84.78 & -28.76 \\
\hline & 318 & $6.91 \times 10^{4}$ & & 84.79 & -29.46 \\
\hline \multirow{3}{*}{$\lambda_{e x}=280 \mathrm{~nm}$} & 298 & $6.73 \times 10^{4}$ & \multirow{3}{*}{-2.78} & 83.11 & -27.54 \\
\hline & 310 & $6.47 \times 10^{4}$ & & 83.14 & -28.55 \\
\hline & 318 & $6.25 \times 10^{4}$ & & 83.08 & -29.20 \\
\hline \multirow{3}{*}{$\lambda_{e x}=295 \mathrm{~nm}$} & 298 & $5.44 \times 10^{4}$ & \multirow{3}{*}{-2.60} & 81.93 & -27.02 \\
\hline & 310 & $5.13 \times 10^{4}$ & & 81.78 & -27.95 \\
\hline & 318 & $5.09 \times 10^{4}$ & & 81.92 & -28.65 \\
\hline
\end{tabular}

\subsection{Hill's Coefficient of BSA-RHB System}

According to the Hill's coefficient of BSA-RHB system, we can make a quantitative analysis for cooperative binding between protein and ligands on the basis of the following Eq. (5) [23]:

$$
\lg \{Y /(1-Y)\}=\lg K_{a}+n_{H} \lg [L]
$$

Where $K_{a}$ is the binding constant, $Y$ is the fractional binding saturation, and $n_{H}$ is the Hill's coefficient. Hill's coefficient is greater than 1 , which exhibits positive cooperativity and its role is enhanced with increasing $n_{H}$. Conversely, Hill's coefficient is less than 1 , which exhibits negative cooperativity and its role is enhanced by decreasing $n_{H}$. A coefficient of 1 indicates non-cooperative reaction.

For fluorescence measurement:

$$
\begin{gathered}
Y /(1-Y)=Q /\left(Q_{m}-Q\right) \\
Q=1-I / I_{0}
\end{gathered}
$$

Where $1 / Q_{m}$ is intercept of the plot $1 / Q$ versus $1 /[L]$. According to the formula (6), Hill's coefficient of BSA-RHB system can be gained from the slope of the plot of log $[Y /$ $(1-Y)]$ versus $\log [L]$. The results were presented in Table 4 . We could see that the values of $n_{H}$ were both equal to 1 approximately at different temperatures by synchronous fluorescence method and fluorescence quenching method, which indicated that there was non-cooperative reaction between BSA and RHB. Meanwhile, it illustrated the synchronous fluorescence method could replace the fluorescence quenching method to determine the cooperative

\begin{tabular}{|c|c|c|c|c|c|c|c|c|}
\hline \multirow{2}{*}{$T / \mathbf{K}$} & \multicolumn{2}{|c|}{$\lambda_{e x}=280 \mathrm{~nm}$} & \multicolumn{2}{|c|}{$\lambda_{e x}=295 \mathrm{~nm}$} & \multicolumn{2}{|c|}{$\Delta \lambda=15 \mathrm{~nm}$} & \multicolumn{2}{|c|}{$\Delta \lambda=60 \mathrm{~nm}$} \\
\hline & $n_{H}$ & $r_{5}$ & $n_{H}$ & $r_{5}$ & $n_{H}$ & $r_{5}$ & $n_{H}$ & $r_{5}$ \\
\hline 298 & 1.08 & 0.9913 & 1.05 & 0.9952 & 1.01 & 0.9963 & 1.03 & 0.9950 \\
\hline 310 & 0.99 & 0.9972 & 1.01 & 0.9952 & 1.05 & 0.9945 & 0.99 & 0.9973 \\
\hline 318 & 1.01 & 0.9990 & 1.01 & 0.9988 & 1.04 & 0.9991 & 1.01 & 0.9994 \\
\hline
\end{tabular}
binding between RHB and BSA.

Table 4. Hill coefficient of BSA-RHB system at different temperatures.

\subsection{Binding Distances Between BSA-RHB System}

According to Förster's non-radiative energy transfer theory, the distance $(r)$ between the donor and the acceptor, critical energy transfer distance $R_{0}$ when the transfer efficiency is $50 \%$, and the energy-transfer efficiency $E$ can be calculated by the formulas [24]:

$$
\begin{gathered}
E=1-F / F_{0}=R_{0}{ }^{6} /\left(R_{0}{ }^{6}+r^{6}\right) \\
R_{0}{ }^{6}=8.79 \times 10^{-25} K^{2} \Phi N^{-4} J \\
J=\sum F(\lambda) \varepsilon(\lambda) \lambda^{4} \Delta \lambda / \sum F(\lambda) \Delta \lambda
\end{gathered}
$$

Where $F_{0}$ is the fluorescence intensity of donor, $F$ is the fluorescence intensity for the donor in presence of same concentration acceptor, $K^{2}$ is the orientation factor, $\Phi$ is the fluorescence quantum yield of the donor in the absence of acceptor, $N$ is a refractive index of the medium, $F(\lambda)$ is the fluorescence intensity of the fluorescence donor at wavelength $\lambda$ and $\varepsilon(\lambda)$ is the molar absorption coefficient of the acceptor at this wavelength. Under these experimental conditions, it has been reported that $K^{2}=2 / 3, N=1.336$ and $\Phi=0.118$ [25]. $J$ is the overlap integral between the fluorescence emission spectrum of the donor and the absorption spectrum of the acceptor. Thus $J, E, R_{0}$ and $r$ were calculated and shown in Table 5. As seen in Table 5, the donor-to-acceptor distance $r<7 \mathrm{~nm}$ indicated that the energy transfer from BSA to RHB occurred with high possibility [26]. The distance $r$ increased and the energy efficiency $E$ decreased with increasing temperature, which resulted in the reduced stability of the binary systems and the values of $K_{a}$. Moreover, the value of $r$ was greater than $R_{0}$ in this study which suggested that RHB could strongly quench the intrinsic fluorescence of BSA by a static quenching mechanism [27]. In addition, from Table 5, the data obtained using the synchronous fluorescence spectrometry $(\Delta \lambda=60 \mathrm{~nm})$ and fluorescence quenching method were basically consistent, 
and the conclusions also were the same. So we could take advantage of synchronous fluorescence $(\Delta \lambda=60 \mathrm{~nm})$ to calculate the combined distance of BSA-RHB.

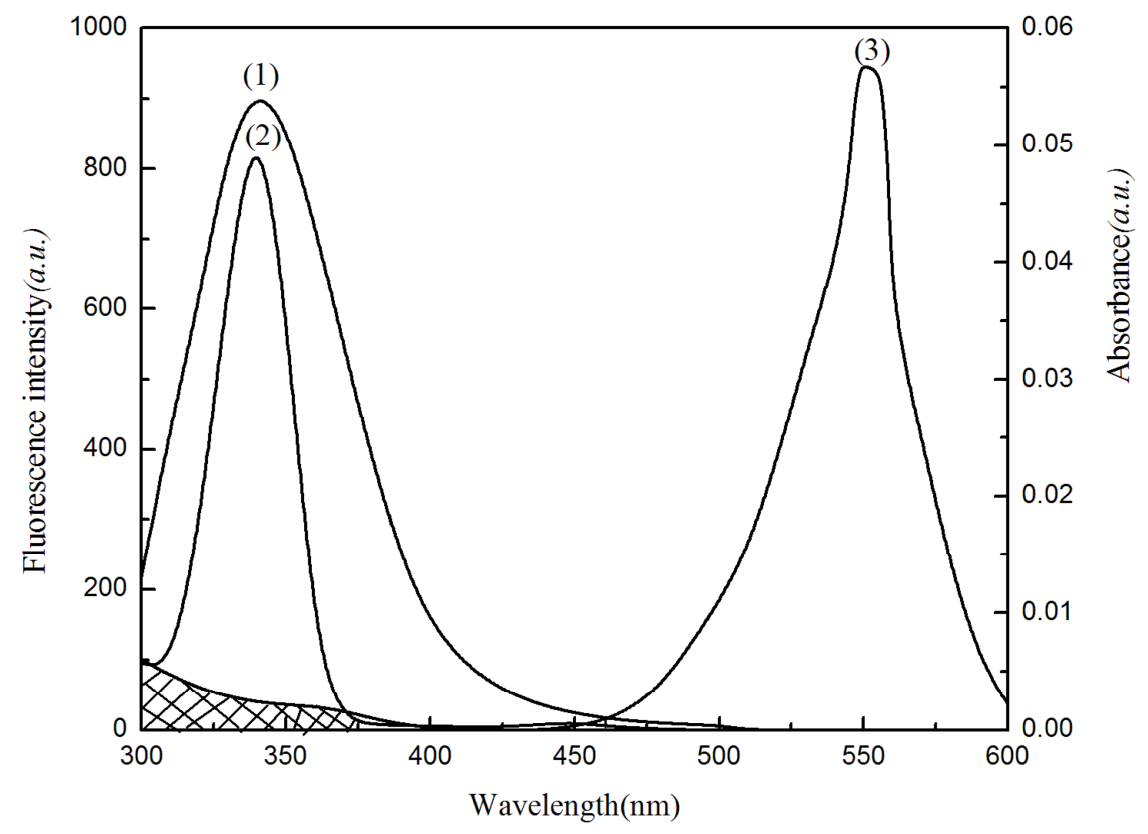

Figure 6. Fluorescence emission spectra (1) and Synchronous fluorescence spectra (2) for BSA and UV absorbance spectra for $R H B$ (3) $T=298 K$; $C_{B S A}=C_{R H B}$ $=4.0 \times 10^{-7} \mathrm{~mol} / \mathrm{L}$.

Table 5. Parameters of $E, J, r, R_{0}$ between $B S A$ and $R H B$ at different temperatures.

\begin{tabular}{|c|c|c|c|c|c|c|c|c|}
\hline system & $\lambda_{e x}=280 \mathrm{~nm}$ & & & & $\Delta \lambda=60 \mathrm{~nm}$ & & & \\
\hline$T / \mathbf{K}$ & $E(\%)$ & $J /\left(\mathrm{cm}^{3} \cdot \mathbf{M}^{-1}\right)$ & $R_{0} / \mathrm{nm}$ & $r / \mathbf{n m}$ & $E(\%)$ & $J /\left(\mathrm{cm}^{3} \cdot \mathbf{M}^{-1}\right)$ & $R_{0} / \mathrm{nm}$ & $r / \mathbf{n m}$ \\
\hline 298 & 2.72 & $8.72 \times 10^{-15}$ & 2.39 & 4.34 & 3.46 & $8.53 \times 10^{-15}$ & 2.38 & 4.14 \\
\hline 310 & 1.71 & $7.12 \times 10^{-15}$ & 2.31 & 4.54 & 2.7 & $6.99 \times 10^{-15}$ & 2.31 & 4.17 \\
\hline 318 & 1.34 & $6.22 \times 10^{-15}$ & 2.26 & 4.63 & 1.99 & $5.21 \times 10^{-15}$ & 2.20 & 4.21 \\
\hline
\end{tabular}

\section{Conclusions}

This paper studied the interaction mechanism between RHB and BSA, and compared the traditional fluorescence quenching method and synchronous fluorescence spectrometry. The results showed that the data of two methods were in the same order of magnitude, and were very close to each other. Additionally, the quenching mechanism and type of interaction force were consistent, which indicated that synchronous fluorescence spectrometry could study the binding mechanism between ligands and protein, and was a useful supplement to the conventional method. Moreover, synchronous fluorescence has some advantages such as good selectivity, high sensitivity, simplified spectrum, narrowed bands and less interference, which makes it more useful than conventional fluorescence quenching in studying the reaction mechanism of ligands with proteins. Study on the mechanism of RHB with BSA is beneficial to further understand the toxicity of RHB to human body, and strengthen the consciousness of food safety.

\section{Acknowledgements}

The authors gratefully acknowledge the financial support of
National Science Foundation of China (Grant No. 20675024) and Hebei Provincial Key Basic Research Program (Grant No. 10967126D).

\section{References}

[1] S. Millan, L. Satish, and S. Kesh, J. Photoch. Photobio., 2016, 162, 248-257.

[2] Z. L. Yang, S. Chen, and Y. X. Zhao, Sensor. Actuat. B-Chem., 2018, 266, 422-428.

[3] J. Ji, Y. Liu, and X. Y. Yang, J. Environ. Manage., 2018, 218, 300-308.

[4] X. R. Li, Y. B. Hao, J. Mol. Struct., 2015, 1091, 109-117.

[5] S. T. Duan, B. S. Liu, and T. T. Li, J. Appl. Spectrosc+., 2017, 84, 411-418.

[6] G. X. Li, B. S. Liu, and Q. J. Zhang, Lumin., 2016, 31, 1054-1062.

[7] P. Zhao, G. Gao, and L. J. Zhang, J. Pharmaceut. Biome., 2017, 141, 262-269.

[8] F. A. Qais, I. Ahmad, J. Pharmaceut. Biome., 2018, 149, 193-205.

[9] G. Rabbani, M. H. Baig, and A. T. Jan, Int. J. Biol. Macromol., 2017, 105, 1572-1580. 
[10] Z. J. Li, Z. C. Wang, and N. Wang, J. Pharmaceut. Biome., 2018, 149, 290-295.

[11] A. Kaur, I. A. Khan, and P. K. Banipal, Spectrochim. Acta. A., 2018, 191, 259-270.

[12] Z. Y. Tian, F. L. Zang, and W. Luo, J. Photochem. Photobiol. B., 2015, 142, 103-109.

[13] J. E. Ali, P. A. Vahid, Food Chem., B. 2016, 202, 426-431.

[14] Z. B. Wu, L. L. Shen, and J. Lu, Food Biophys., 2017, 12, 78-87.

[15] M. K. Amroabadi, A. Taheri-Kafrani, and L. H. Saremi, Int. J. Biol. Macromol., 2018, 108, 263-271.

[16] H. L. Xu, N. N. Yao, and G. Y. Li, Spectrosc. Lett., 2014, 47, 119-130.

[17] S. N. Cao, B. S. Liu, and G. X. Li, J. Lumin., 2014, 145, 94-99.

[18] M. Makarska-Bialokoz, Spectrochim. Acta. A., 2016, 193, 23-32.

[19] G. X. Li, B. S. Liu, and Q. J. Zhang, Lumin., 2016, 31, 1054-1062.
[20] C. B. Xu, J. L. Gu, and X. P. Ma, Spectrochim. Acta. A., 2014, $125,391-395$

[21] S. Huang, H. Qiu, and Y. Liu, Colloid. Surface. B, 2016, 148, 165-172.

[22] M. B. Bolattin, S. T. Nandibewoor, and S. D. Joshi, Ind. Eng. Chem. Res., 2016, 55, 5454-5464.

[23] Q. J. Zhang, B. S. Liu, and R. Han, Spectrosc. Lett., 2016, 49, 208-213.

[24] A. Hasanzadeh, G. Dehghan, and M. Shaghaghi, J. Mol. Liq., 2017, 248, 459-467.

[25] Z. G. Li, J. Q. Wang, and T. Ren, Med. Chem. Res., 2016, 25, 1009-1019.

[26] A. T. Buddanavar, S. T. Nandibewoor, J. Pharmaceut Anal., 2017, 7, 148-155.

[27] F. Moeinpour, F. S. Mohseni-Shahri, and B. Malaekeh-Nikouei, Chem-Biol. Interact., 2016, 257, 4-13. 